\title{
Comparing the effects of dezocine with lidocaine on prevention of propofol injection pain in adults: a prospective randomized double-blind trial
}

\section{Xuqin Zhu}

Fudan University Shanghai Cancer Center

Songlin Jia

Fudan University Shanghai Cancer Center

\section{Yajun Xu}

Fudan University Shanghai Cancer Center

Zhirong Sun ( $\square$ sunzij781101@163.com )

Fudan University Shanghai Cancer Center https://orcid.org/0000-0002-7961-5993

Research article

Keywords: Dezocine, Lidocaine, Propofol injection pain

Posted Date: September 27th, 2019

DOI: https://doi.org/10.21203/rs.2.9717/v3

License: @ (i) This work is licensed under a Creative Commons Attribution 4.0 International License. Read Full License 


\section{Abstract}

Background Propofol injection pain (PIP) is common and may decrease patient comfort. The aim of this study was to compare the effects of dezocine with lidocaine on prevention of PIP.Methods 235 patients, who scheduled for elective surgery, aged 18-80 years and American Society of Anesthesiologists (ASA) I or II, were randomly assigned into five groups ( $n=47$ each). All patients were induced through the dorsal hand vein or antecubital vein. The five groups were given the following medication intravenously: saline (Group I), lidocaine $20 \mathrm{mg}$ (Group II), lidocaine $40 \mathrm{mg}$ (Group III), dezocine $2 \mathrm{mg}$ (Group IV) and dezocine $4 \mathrm{mg}$ (Group V). Twenty seconds later all patients received a propofol infusion and were asked to grade pain or discomfort in the hand or forearm according to a four-point scale until anesthesia.Results Three groups showed a significantly lower incidence of total PIP than Group I: Group III (OR [Odds ratio]: 0.39 $(0.16,0.93))$; Group IV (OR: $0.21(0.09,0.51))$ and Group V (OR: $0.12(0.05,0.30))$. Group IV showed a significantly lower incidence of total PIP than Group II (OR: $0.32(0.13,0.77))$. Group V showed a significantly lower incidence of total PIP than Group II (OR: $0.15(0.06,0.39))$ or Group III (OR: $0.30(0.13$, 0.72)).Conclusion Dezocine is a novel agent to alleviate PIP and appears to some extent to be more effective than lidocaine.

\section{Background}

Propofol is a widely-used intravenous anesthetic agent for the induction and maintenance of general anesthesia and for moderate to deep sedation, due to its rapid onset of action and fast recovery process. However, propofol injection pain (PIP) is common in clinical practice and has been ranked seventh among the 33 most important low-morbidity clinical outcomes by a panel of expert anesthesiologists [1]. The overall incidence of PIP and high intensity PIP have been found to be $28-90 \%$ and $38 \%$, respectively, during the course of induction [2-4]. Although PIP is not a very serious problem, it can be an unpleasant sensation for some patients and lead to a fear of anesthesia.

Previous studies have assessed various methods to alleviate PIP, including changing the injection speed, warming up propofol, choosing a large vein and the addition of some drugs [5-13]. At present, lidocaine is the most common drug used to relieve PIP. However, even if pretreatment with lidocaine or lidocaine mixed with propofol were given, the overall incidence of PIP and high-intensity PIP were still as high as $30.2 \%$ and $11.8 \%$, respectively [4]. Regardless, this study proposes to demonstrate the potential of dezocine for attenuating PIP and improvement of patient satisfaction with induction of anesthesia.

Dezocine is widely used as perioperative analgesic drug in China. It is a mixed agonist-antagonist opioid, a full agonist of $k$-receptors and a partial agonist of $\mu$-receptors $[14,15]$. The analgesic effect of dezocine originates mainly from its binding to $\mathrm{k}$-receptors. The aim of this study was to investigate the effects of different doses of dezocine on prevention of PIP compared with lidocaine.

\section{Methods}




\section{Ethics}

This prospective randomized, double-blind trial was approved by Fudan University, Shanghai, Cancer Center Institutional Human Ethics Committee (Protocol number: 1703170-14) on April 5th, 2017. The trial was registered in the Chinese Clinical Trial Registry (ChiCTR.org ID: ChiCTR-IPR-17011234).

\section{Study protocol}

After providing written informed consent, 235 patients were included in the trial. The sample size was based on previously-published studies $[4,16]$. The incidence rates of PIP in the control group, lidocaine pretreatment group and dezocine pretreatment group in these studies were $64 \%, 30 \%$ and $28 \%$, respectively. The incidence of PIP in the lidocaine pretreatment group was closer to the control group. 42 patients per group would be required to provide the study with $90 \%$ power with a significance level of $5 \%$ $(\beta=0.9$ and $a=0.05)$. Assuming a dropout rate of $10 \%, 47$ patients were enrolled in each group.

Patients were American Society of Anesthesiologists physical status I-II, aged 18-80 years and scheduled for elective surgery induced through the dorsal hand vein or antecubital vein. Exclusion criteria included a history of hepatic or renal insufficiency, severe neurological or cardiovascular disease, psychological disease or cognitive disorder, obesity or difficult airway, peripheral phlebitis, drug abuse or opioid therapy for chronic pain, allergy to dezocine, lidocaine and other drugs, pregnancy and difficulty in communicating well.

Patients were randomly assigned into five groups using computer-generated codes. Group I received saline $2 \mathrm{ml}$; Group II received lidocaine 20 mg; Group III received lidocaine 40 mg; Group IV received dezocine $2 \mathrm{mg}$ and Group $\mathrm{V}$ received dezocine $4 \mathrm{mg}$. The doses of lidocaine were chosen according to previous studies $[16,17]$. The medication given to patients in Groups II-V was diluted to $2 \mathrm{ml}$ with saline. The study was performed by three blinded investigators. The first investigator prepared each test medication. The second investigator, who was blind to the type of test medication, performed induction of general anesthesia. The third investigator, who was also blind to the type of test medication, evaluated pain score and recorded relevant information.

Before entering the operating room, a 20-gauge cannula was placed into each patient's dorsal hand vein or antecubital vein. Upon arrival, each patient received routine monitoring including non-invasive arterial pressure, electrocardiography and pulse oximetry. Patients were then given medication or placebo, and 20 seconds later were given the propofol infusion (target-controlled infusion plasma concentration: 3-4 $\mu \mathrm{g} / \mathrm{ml}$ ). Patients were asked to grade pain in the hand or forearm according to a four-point scale (none, mild, moderate, severe) during propofol infusion until anesthesia. The infusion time was divided into two periods: early period (from 0 to 10th second) and late period (from 11th second to anesthesia). Just after anesthesia was induced, sufentanil and muscle relaxant were administered and anesthesia proceeded as normal.

\section{Statistical analysis}


All statistical analyses were performed with SPSS v 18.0 software package, and forest plots were generated with Stata $v$ 14.0. Continuous variables were described as mean \pm SD (standard deviation), and count variables were described as frequency and percentage. A T test, ANOVA or non-parametric test was used to compare continuous variables, while count variables were compared using a $\chi^{2}$ or Fisher exact test. Odds ratios (ORs) were used to compare the incidence of PIP among different groups and were adjusted, while baseline information was not balanced. All tests were two-sided and 0.05 was set as the $P$ value for significance.

\section{Results}

Of the 245 enrolled patients, ten were excluded from the study due to failure to insert into the patients' dorsal hand vein or antecubital vein or difficulty in communicating well with investigators. Therefore, 47 patients in each group were included and subjected to further statistical analysis (Fig. 1).

No significant differences were observed between groups in terms of sex, height, weight, BMI (body mass index) and IV (intravenous injection) placement. However, there was a significant difference in age between the five groups (Table 1).

\section{The effects of dezocine on prevention of total propofol injection pain}

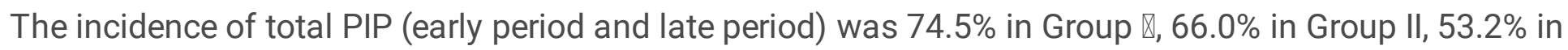

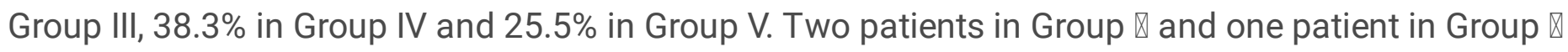
experienced severe pain. No severe PIP was observed in the other three groups (Table 2). Groups III, IV and V showed significantly lower incidence of total PIP than Group I. Group IV showed significantly lower incidence of total PIP than Group II. Group V showed significantly lower incidence of total PIP than Groups II and III (Fig. 2).

\section{The effects of dezocine on prevention of early propofol injection pain}

Groups III, IV and V showed significantly lower incidence of early PIP than Groups I and II (Table 3, Fig. 3).

\section{The effects of dezocine on prevention of late propofol injection pain}

Groups IV and V showed significantly lower incidence of late PIP than Group I. Groups IV and V showed significantly lower incidence of late PIP than Groups II and III, respectively (Table 4, Fig. 4).

\section{Discussion}

Propofol can irritate nociceptive receptors or free nerve endings in the peripheral veins, causing pain or discomfort. The direct irritation of the intima by propofol can stimulate the production of bradykinin [18]. Bradykinin is a potent inflammatory factor which can cause vasodilation and hyperpermeability of veins, which in turn increases exposure of the free nerve endings to propofol. PIP lasts for the duration of 
propofol infusion: one previous paper stated that pain during the early stage was due to direct stimulation of propofol in the veins, while the later pain was due to local generation of bradykinin [19].

Lidocaine is not only commonly used to alleviate PIP, but is also used as a comparator when studying a new drug to relieve PIP $[2,5,9,11,16,19]$. Therefore, we also chose lidocaine as a comparison to study dezocine and chose the doses of lidocaine according to previous studies. In this study we found that just $40 \mathrm{mg}$ lidocaine could significantly reduce the incidence of total PIP and early PIP, suggesting that its mode of action is blocking the peripheral nerve pathway to produce short-acting local anesthesia. Therefore, lidocaine showed no significant effect on late PIP. Moreover, 10 patients experienced moderate PIP and one patient experienced severe PIP, even when pretreated with lidocaine. Our results also showed that the effect of $40 \mathrm{mg}$ lidocaine on early PIP was statistically better than that of $20 \mathrm{mg}$ lidocaine; therefore, we suppose that larger doses of lidocaine will be more effective to reduce PIP and we will do further study with larger doses of lidocaine in the future.

In our previous pilot study, pretreatment with $1 \mathrm{mg}$ dezocine did not significantly reduce PIP. Therefore, we investigated PIP at larger doses of dezocine in the present study. Our results demonstrated that $2 \mathrm{mg}$ and $4 \mathrm{mg}$ dezocine could significantly reduce the incidence of PIP in both early and late periods and there was no significant difference between the effect of $2 \mathrm{mg}$ and $4 \mathrm{mg}$ dezocine on PIP. No patient experienced severe PIP. This finding is similar to a previous study, which also found that $2 \mathrm{mg}$ dezocine could reduce PIP [16]. However, our study differed in that we studied dezocine at two different doses and propofol infusion at two different time periods. It is useful for us to investigate the mechanisms of how dezocine and lidocaine prevent PIP.

Other previous studies reported that other opioids (remifentanil, alfentanil, fentanil, pethidine) could also alleviate PIP $[2,11,20,21]$, but the precise mechanisms of how opioids prevent PIP remain unclear. One previous study [22] found no evidence that opioid (alfentanil) application with a tourniquet could relieve pain, suggesting that the effects of opioids on prevention of PIP may be via central, but not peripheral, opioid receptors. However, other studies showed that administration of other opioids (remifentanil, alfentanil) without a tourniquet could alleviate PIP [9, 20,21]. Dezocine injection was performed without a tourniquet in our study, and we assumed that its effect on PIP was more likely to be mediated via central opioid receptors.

As shown in Fig. 3, either $2 \mathrm{mg}$ or $4 \mathrm{mg}$ dezocine could eliminate early PIP significantly more effectively than $20 \mathrm{mg}$ lidocaine. We also found that $2 \mathrm{mg}$ or $4 \mathrm{mg}$ dezocine could alleviate late PIP significantly more effectively than $20 \mathrm{mg}$ or $40 \mathrm{mg}$ lidocaine, respectively. In terms of total PIP, the relieving effect of 4 $\mathrm{mg}$ dezocine was better than $20 \mathrm{mg}$ or $40 \mathrm{mg}$ lidocaine, respectively. Our study also showed that the incidence of total PIP was $25.5 \%$ in the $4 \mathrm{mg}$ dezocine group, compared to $53.2 \%$ in the $40 \mathrm{mg}$ lidocaine group. Although dezocine could not completely eliminate PIP, its effect on prevention of PIP appeared to be better than that of lidocaine. It is well worth mentioning that not everyone is fit to receive lidocaine for prevention of PIP due to local anesthetic allergy or arrhythmia; in this population, dezocine may be an effective agent in managing PIP. 
Other effective non-pharmacological approaches, or combinations of pharmacological approaches, have been recommended to relieve PIP including warming propofol, venous occlusion with a tourniquet, applying microfiltration and changing the lipid composition $[3,12,17,23,24]$. However, no method could eliminate PIP completely and most of these methods were not routinely available in clinical practice, except venous occlusion with a tourniquet.

There are some potential limitations in our study. First, although our results showed that $2 \mathrm{mg}$ or $4 \mathrm{mg}$ dezocine could reduce the incidence of PIP, it could not completely eliminate PIP. Future studies could investigate larger doses of dezocine; however, we should also consider the side effects of dezocine with increasing dose. We did not collect information about the effects of dezocine on analgesia and its side effects post-operation. Second, there was a significant difference in age among the five groups, although patients were randomly assigned using computer-generated codes. Therefore, ORs were used to compare the incidence of PIP among the different groups and were adjusted while baseline information was not balanced. Third, we chose just 20 or $40 \mathrm{mg}$ lidocaine as a comparison; future studies need to consider larger doses of lidocaine. Fourth, there was a lack of correction when we made multiple comparisons. Because this was an exploratory study, we will do further study with larger samples of patients for validation in the future.

\section{Conclusions}

Although the precise mechanisms of how dezocine prevents PIP are not yet clear, our study has shown that it is a potentially effective agent to alleviate PIP, and it appears to some extent to be more effective than lidocaine.

\section{List Of Abbreviations}

PIP: Propofol injection pain; ASA: American Society of Anesthesiologists; SD: standard deviation; OR: Odds ratio; BMI: body mass index; Cl: confidence interval; IV: intravenous injection.

\section{Declarations}

\section{Ethics approval and consent to participate}

This study was approved by Fudan University, Shanghai, Cancer Center Institutional Human Ethics Committee. Written informed consent was obtained from all subjects. The study was registered in the Chinese Clinical Trial Registry (ChiCTR.org ID: ChiCTR-IPR-17011234). Initial registration date was 04/24/2017.

\section{Consent for publication}

Not applicable. 
Availability of data and material

The datasets used and/or analyzed during the current study are available from the corresponding author on reasonable request.

\section{Competing interests}

The authors declare that they have no competing interests.

\section{Funding}

This study was supported by Shanghai Municipal Commission of Health and Family Planning Foundation [grant number 201540080].

\section{Authors' contributions}

XQZ, SLJ and ZRS conceived the study design and drafted the study protocol. XQZ, SLJ, YJX and ZRS all participated in the study design and coordination. XQZ, SLJ and YJX contributed to data collection and data analysis. ZRS was the principal investigator and had overall responsibility for this study. XQZ and SLJ drafted and revised the manuscript. ZRS critically revised the manuscript. All authors have read and approved the final manuscript.

\section{Acknowledgements}

The authors thank Wei Zhang (Department of Health Statistics and Social Medicine, School of Public Health, Fudan University, Shanghai, China) for his contribution to the statistical analysis.

\section{References}

1. Macario A, Weinger $M$, Truong $P$, Lee $M$. Which clinical anesthesia outcomes are both common and important to avoid? The perspective of a panel of expert a Anesth Analg. 1999; 88: 1085-91.

2. Lyons B, Lohan D, Flynn C, McCarroll M. Modification of pain on injection of propofol a comparison of pethidine and lignocaine. Anaesthesia. 1996; 51: 394-5.

3. Mangar D, Holak EJ. Tourniquet at $50 \mathrm{~mm} \mathrm{Hg}$ followed by intravenous lidocaine diminishes hand pain associated with propofol injection. Anesth Analg. 1992; 74: 250-2.

4. Euasobhon P, Dej-Arkom S, Siriussawakul A, Muangman S, Sriraj W, Pattanittum P, et al. Lidocaine for reducing propofol-induced pain on induction of anaesthesia in adults. Cochrane Database Syst Rev. 2016; 2: Cd007874.

5. Goktug A, Gulec H, Takmaz SA, Turkyilmaz E, Basar H. Lidocaine alleviates propofol related pain much better than metoprolol and nitroglycerin. Braz J Anesthesiol. 2015; 65: 338-42.

6. Shimizu T, Inomata S, Kihara S, Toyooka H, Brimacombe JR. Rapid injection reduces pain on injection with propofol. Eur J Anaesthesiol. 2005; 22: 394-6. 
7. Grauers A, Liljeroth E, Akeson J. Propofol infusion rate does not affect local pain on Acta Anaesthesiol Scand. 2002; 46: 361-3.

8. Seki S, Sekine R, Aketa K, Kobayashi Y, Ichimiya T, Tsuchida $H$, et al. Induction of anesthesia with propofol injected through a central venous catheter. Masui. 1999; 48: 62-6.

9. Nathanson MH, Gajraj NM, Russell JA. Prevention of pain on injection of propofol: a comparison of lidocaine with alfentanil. Anesth Analg. 1996; 82: 469-71.

10. Batra YK, Qattan ARA, Marzouk HM, Smilka M, Agzamov A. Ketamine pretreatment with venous occlusion attenuates pain on injection with propofol. Eur J Anaesthesiol. 2005; 22: 69-70.

11. Pang WW, Mok MS, Huang S, Hwang MH. The analgesic effect of fentanyl, morphine, meperidine, and lidocaine in the peripheral veins: a comparative study. Anesth Analg. 1998; 86: 382-6.

12. Youn AM, Hsu TM. Heated carrier fluids in decreasing propofol injection pain: a randomized, controlled trial. Korean J Anesthesiol. 2017; 70: 33-8.

13. Sargin M, Uluer MS, Aydogan E. Hyoscine N-butylbromide for preventing propofol injection pain: a randomized, placebo-controlled and double-blind s Med Princ Pract. 2018; 27: 39-43.

14. O'Brien JJ, Dezocine BP. A preliminary review of its pharmacodynamic and pharmacokinetic properties, and therapeutic efficacy. Drugs. 1989; 38: 226-48.

15. Downing JW, Brock-Utne JG, Barclay A, Schwegmann IL. WY 16225 (dezocine), a new synthetic opiate agonist-antagonist and potent analgesic: comparison with morphine for relief of pain after lower abdominal surgery. Br J Anaesth. 1981; 53: 59-64.

16. Lu Y, Ye Z, Wong GT, Dong C, Yu J. Prevention of injection pain due to propofol by dezocine: a comparison with lidocaine. Indian J Pharmacol. 2013; 45: 619-21.

17. Zirak N, Bameshki A, Yazdani M, Gilani MT. Lipid composition and lidocaine effect on immediate and delayed injection pain following propofol administration. Anesth Essays Res. 2016; 10: 29-32.

18. Ambesh SP, Dubey PK, Sinha PK. Ondansetron pretreatment to alleviate pain on propofol injection: a randomized, controlled, double-blinded study. Anesth Analg. 1999; 89: 197-9.

19. Asik I, Yorukoglu D, Gulay I, Tulunay M. Pain on injection of propofol: comparison of metoprolol with lidocaine. Eur J Anaesthesiol. 2003; 20: 487-9.

20. Chae YJ, Min SK, Park SK, Kim SM, Won YJ, Cho HB. Reduction of microemulsion propofol-induced injection pain via target-controlled remifentanil infusion. J Int Med Res. 2011; 39: 2151-7.

21. Fletcher JE, Seavell CR, Bowen DJ. Pretreatment with alfentanil reduces pain caused by propofol. $\mathrm{Br}$ J Anaesth. 1994; 72: 342-4.

22. Wrench IJ, Girling KJ, Hobbs GJ. Alfentanil-mediated analgesia during propofol injection: no evidence for a peripheral action. Br J Anaesth. 1996; 77: 162-4.

23. Picard P, Tramer MR. Prevention of pain on injection with propofol: a quantitative systematic review. Anesth Analg. 2000; 90: 963-9.

24. Davies AF, Vadodaria B, Hopwood B, Dexter T, Conn D. Efficacy of microfiltration in decreasing propofol-induced pain. Anaesthesia. 2002; 57: 557-61. 


\section{Tables}

Table 1. Demographic data

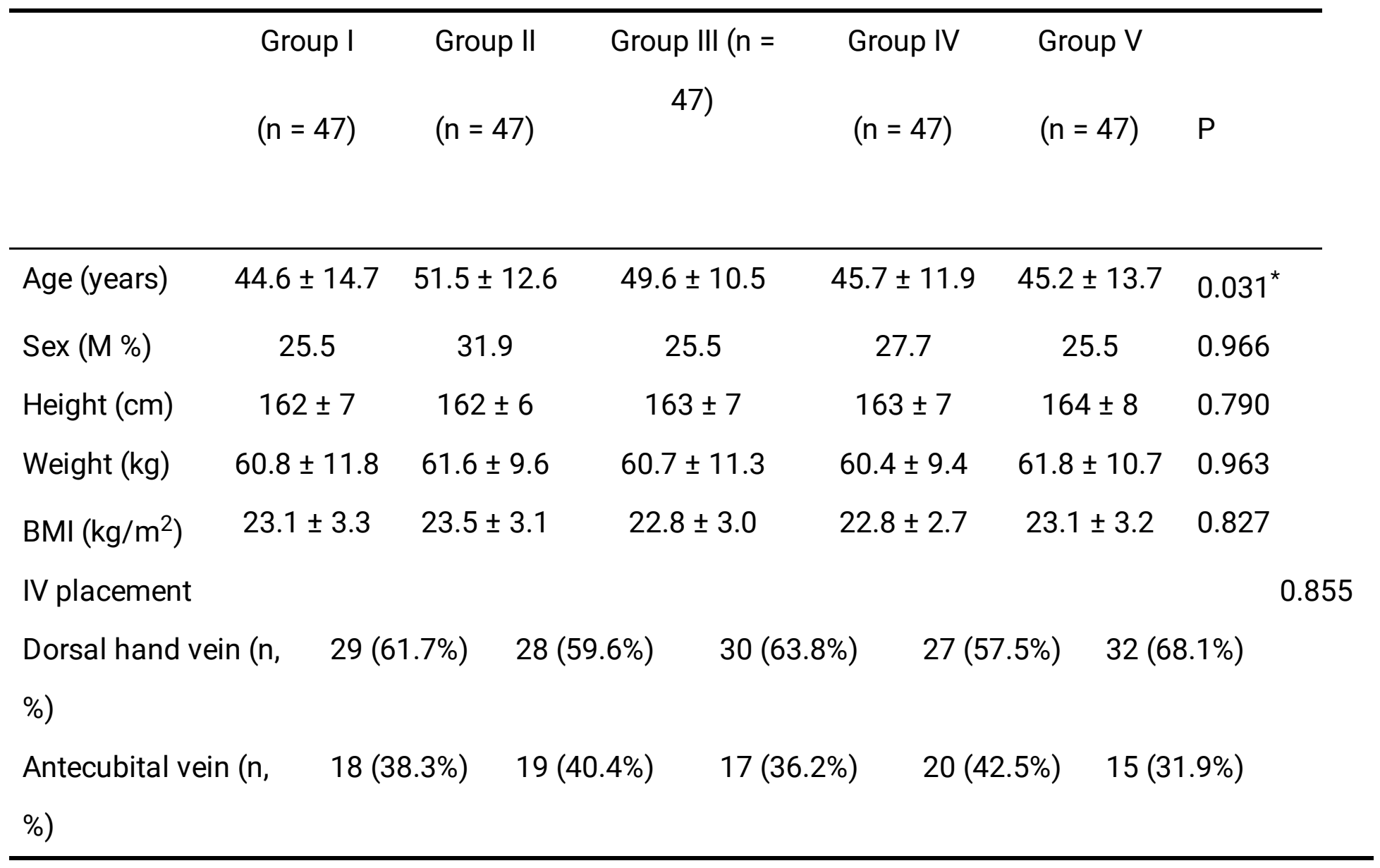

Values are described as mean \pm SD (standard deviation) or percentage (\%) of patients. No significant differences were observed between groups in sex, height, weight, BMI (body mass index) and IV (intravenous injection) placement. Significant difference in age was observed between groups.

Table 2 Total propofol injection pain and its severity in the five groups 


\begin{tabular}{cccccc}
\hline Group & None & Pain & Mild & Moderate & Severe \\
& $(n, \%)$ & $(n, \%)$ & & & \\
\hline I (n=47) & $12(25.5 \%)$ & $35(74.5 \%)$ & 24 & 9 & 2 \\
II (n= 47) & $16(34.0 \%)$ & $31(66.0 \%)$ & 25 & 5 & 1 \\
III (n= 47) & $22(46.8 \%)$ & $25(53.2 \%)$ & 20 & 5 & 0 \\
IV (n= 47) & $29(61.7 \%)$ & $18(38.3 \%)$ & 18 & 0 & 0 \\
V (n= 47) & $35(74.5 \%)$ & $12(25.5 \%)$ & 12 & 0 & 0 \\
\hline
\end{tabular}

Data are described as frequency and percentage (\%) of patients. The severity of pain was graded as: none, mild, moderate or severe.

Table 3 Early propofol injection pain and its severity in the five groups

\begin{tabular}{cccccc}
\hline Group & None & Pain & Mild & Moderate & Severe \\
& $(n, \%)$ & $(n, \%)$ & & & \\
\hline I (n= 47) & $1327.7 \%)$ & $34(72.3 \%)$ & 23 & 9 & 2 \\
II (n= 47) & $22(46.8 \%)$ & $25(53.2 \%)$ & 21 & 3 & 1 \\
III (n=47) & $32(68.1 \%)$ & $15(31.9 \%)$ & 14 & 1 & 0 \\
IV (n= 47) & $33(70.2 \%)$ & $14(29.8 \%)$ & 14 & 0 & 0 \\
V (n= 47) & $37(78.7 \%)$ & $10(21.3 \%)$ & 10 & 0 & 0 \\
\hline
\end{tabular}

Data are described as frequency and percentage (\%) of patients. The severity of pain was graded as: none, mild, moderate or severe.

Table 4 Late propofol injection pain and its severity in the five groups 


\begin{tabular}{cccccc}
\hline Group & None & Pain & Mild & Moderate & Severe \\
& $(n, \%)$ & $(n, \%)$ & & & \\
\hline I (n= 47 I & $15(31.9 \%)$ & $32(68.1 \%)$ & 22 & 8 & 2 \\
II (n= 47) & $21(44.7 \%)$ & $26(55.3 \%)$ & 21 & 4 & 1 \\
III (n= 47) & $22(46.8 \%)$ & $25(53.2 \%)$ & 20 & 5 & 0 \\
IV (n=47) & $34(72.3 \%)$ & $13(27.7 \%)$ & 13 & 0 & 0 \\
V (n= 47) & $39(83.0 \%)$ & $8(17.0 \%)$ & 8 & 0 & 0 \\
\hline
\end{tabular}

Data are described as frequency and percentage (\%) of patients. The severity of pain was as graded: none, mild, moderate or severe.

\section{Figures}

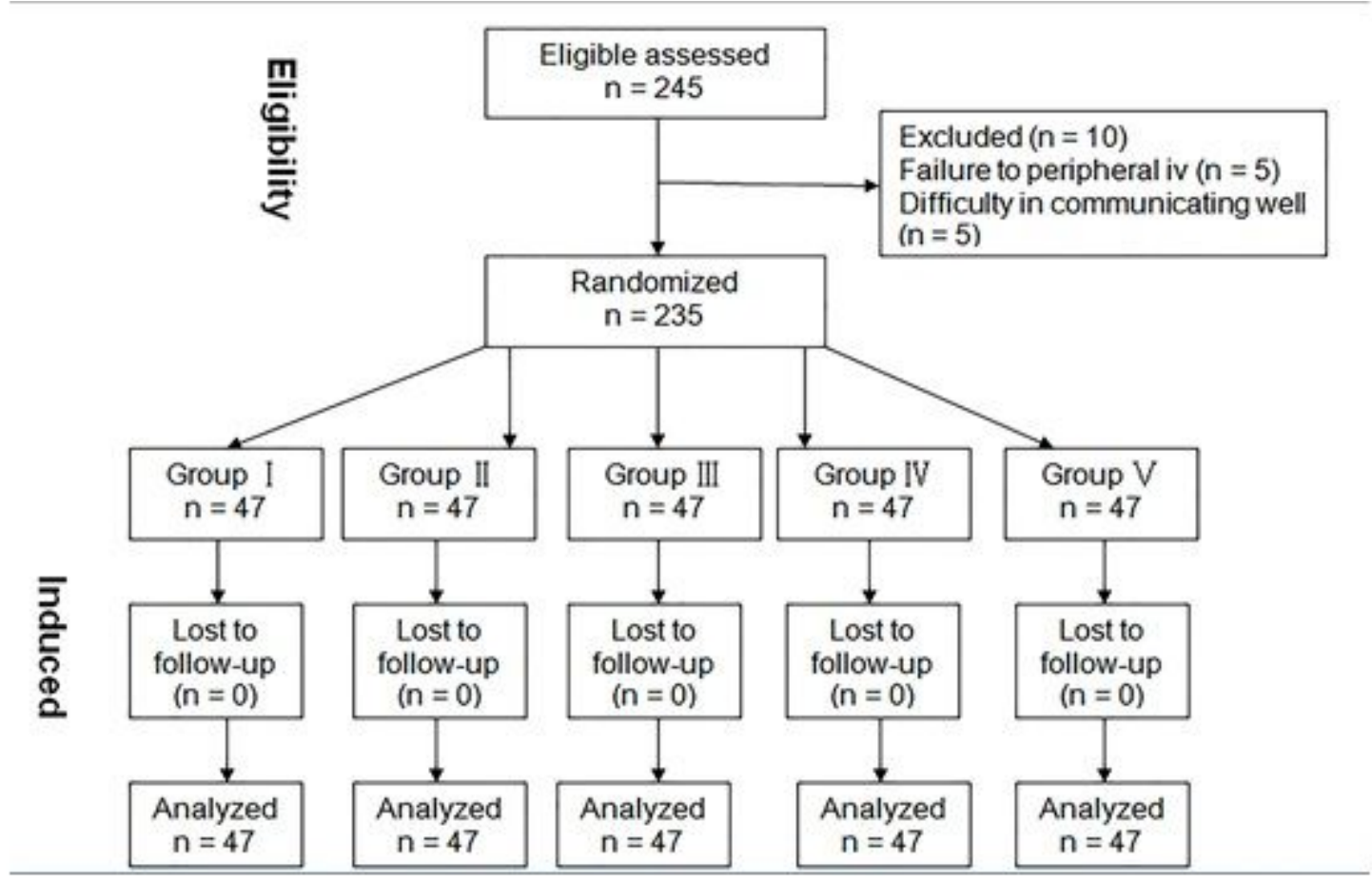

Figure 1

Flow diagram of subjects inclusion and exclusion. 
Group II (Lidocaine 20mg) vs. Group I (Saline)

Group III (Lidocaine 40mg) vs. Group I (Saline)

Group III (Lidocaine 40mg) vs. Group II (Lidocaine 20mg)

Group IV (Dezocine 2mg) vs. Group I (Saline)

Group V (Dezocine 4mg) vs. Group I (Saline)

Group V (Dezocine 4mg) vs. Group IV (Dezocine 2mg)

Group IV (Dezocine 2mg) vs. Group II (Lidocaine 20mg)

Group IV (Dezocine 2mg) vs. Group III (Lidocaine $40 \mathrm{mg}$ )

Group V (Dezocine 4mg) vs. Group II (Lidocaine 20mg)

Group V (Dezocine 4mg) vs. Group III (Lidocaine 40mg)

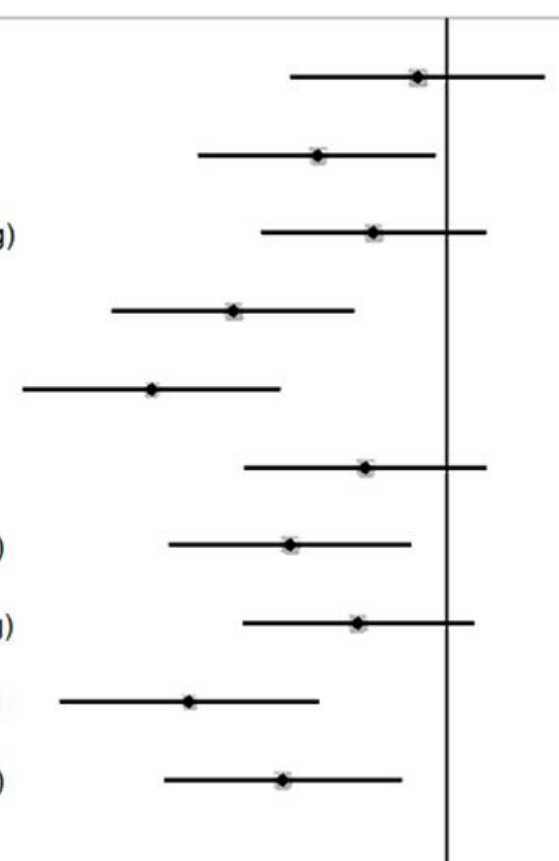

$0.81(0.32,2.04)$

$0.39(0.16,0.93)$

$0.59(0.26,1.34)$

$0.21(0.09,0.51)$

$0.12(0.05,0.30)$

$0.55(0.23,1.34)$

$0.32(0.13,0.77)$

$0.53(0.23,1.22)$

$0.15(0.06,0.39)$

$0.30(0.13,0.72)$

\section{Figure 2}

Comparison of total propofol injection pain among the five groups. $\mathrm{Cl}=$ confidence interval, $\mathrm{OR}=$ odds ratio. 
Group II (Lidocaine 20mg) vs. Group I (Saline)

Group III (Lidocaine 40mg) vs. Group I (Saline)

Group III (Lidocaine 40mg) vs. Group II (Lidocaine 20mg)

Group IV (Dezocine 2mg) vs. Group I (Saline)

Group V (Dezocine 4mg) vs. Group I (Saline)

Group V (Dezocine 4mg) vs. Group IV (Dezocine 2mg)

Group IV (Dezocine 2mg) vs. Group II (Lidocaine 20mg)

Group IV (Dezocine 2mg) vs. Group III (Lidocaine 40mg)

Group V (Dezocine 4mg) vs. Group II (Lidocaine 20mg)

Group V (Dezocine 4mg) vs. Group III (Lidocaine 40mg)
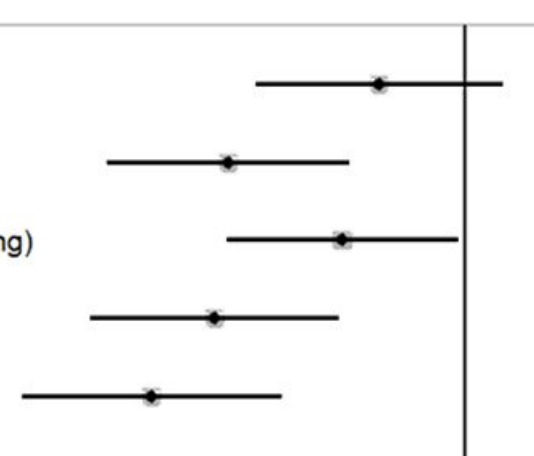

$0.16(0.07,0.40)$

$0.10(0.04,0.26)$

$0.64(0.25,1.63)$

$0.33(0.13,0.80)$

$0.88(0.36,2.16)$

$0.20(0.07,0.51)$

$0.58(0.23,1.45)$

\section{Figure 3}

Comparison of early propofol injection pain among the five groups. $\mathrm{Cl}=$ confidence interval, $\mathrm{OR}=$ odds ratio. 


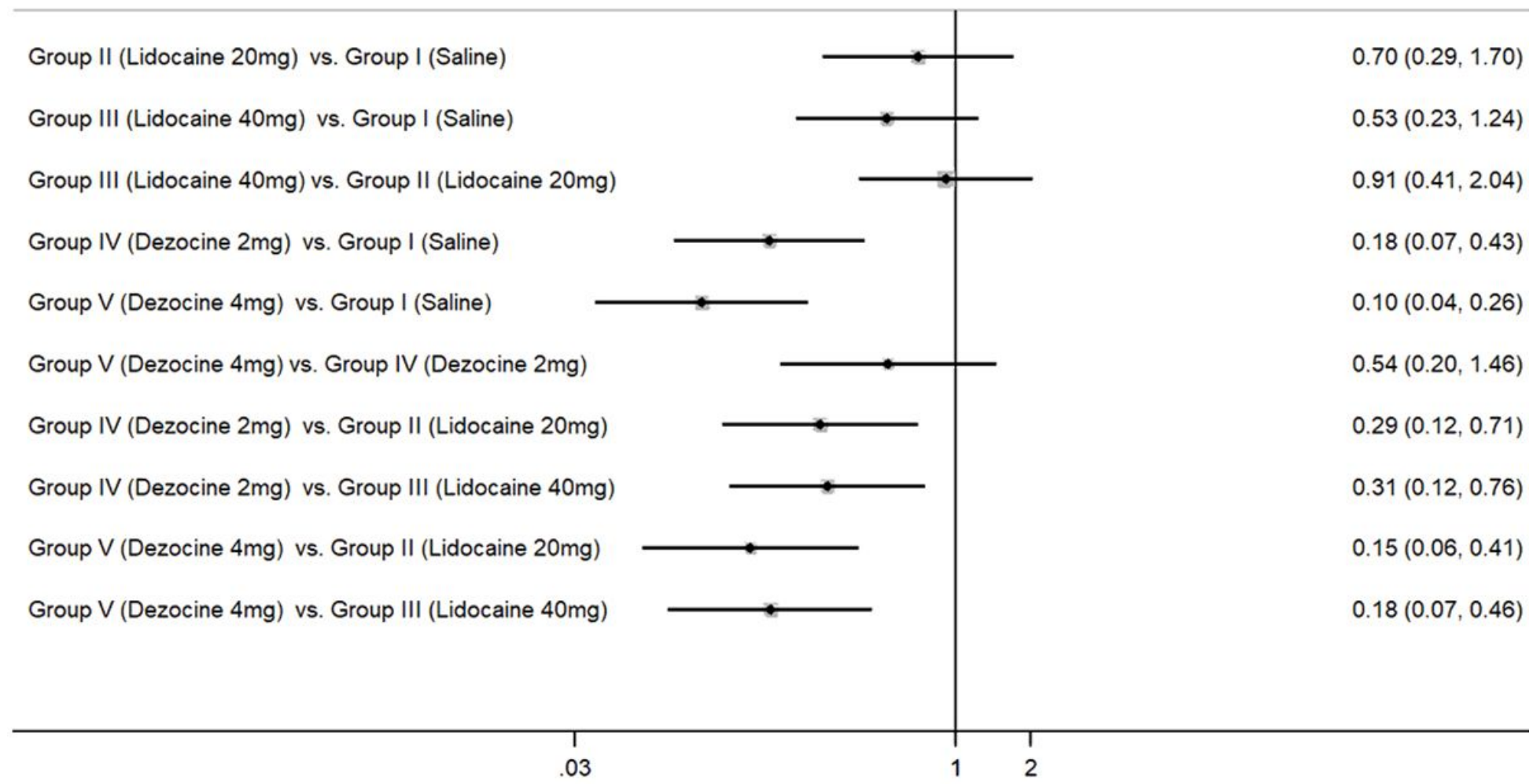

\section{Figure 4}

Comparison of late propofol injection pain among the five groups. $\mathrm{Cl}=$ confidence interval, $\mathrm{OR}=$ odds ratio. 